Regular Article

Tighlighted Leaper selected by Bditor-in-Ghief

\title{
Effect of a Ferric Citrate Formulation, a Phosphate Binder, on Oxidative Stress in Chronic Kidney Diseases-Mineral and Bone Disorder Patients Receiving Hemodialysis: A Pilot Study
}

\author{
Motoko Tanaka, ${ }^{a, \#}$ Shigeyuki Miyamura, ${ }^{b, \#}$ Tadashi Imafuku, ${ }^{b}$ Yuna Tominaga,${ }^{b}$ \\ Hitoshi Maeda, ${ }^{b}$ Makoto Anraku, ${ }^{c}$ Keishi Yamasaki, ${ }^{c}$ Daisuke Kadowaki, ${ }^{d}$ Yu Ishima,${ }^{b}$ \\ Hiroshi Watanabe, ${ }^{b}$ Tomoko Okuda, ${ }^{a}$ Kazuko Itoh, ${ }^{a}$ Kazutaka Matsushita, ${ }^{a}$ Masafumi Fukagawa, ${ }^{e}$ \\ Masaki Otagiri, ${ }^{c}$ and Toru Maruyama*,b \\ ${ }^{a}$ Department of Nephrology, Akebono Clinic; 5-1-1 Shirafuji-machi, Kumamoto 861-4112, Japan: ${ }^{b}$ Department \\ of Biopharmaceutics, Graduate School of Pharmaceutical Sciences, Kumamoto University; 5-1 Oe-honmachi, \\ Kumamoto 862-0973, Japan: ${ }^{c}$ Faculty of Pharmaceutical Sciences, Sojo University; 4-22-1 Ikeda, Kumamoto \\ 860-0082, Japan: 'Faculty of Clinical Pharmaceutical Sciences, Kumamoto University; Kumamoto 862-0973, Japan. \\ and ${ }^{e}$ Division of Nephrology, Endocrinology and Metabolism, Tokai University School of Medicine; Isehara, \\ Kanagawa 259-1193, Japan. \\ Received December 16, 2015; accepted February 29, 2016
}

A ferric citrate formulation for treating hyperphosphatemia is a new therapeutic that not only suppresses the accumulation of phosphorus in patients with chronic kidney disease-mineral bone disorders (CKD-MBD), but also ameliorates anemia caused by iron deficiency. In contrast, it has been demonstrated that intravenous iron injection markedly increases oxidative stress. This study was designed to investigate the effect of a ferric citrate formulation on oxidative stress in CKD-MBD patients receiving hemodialysis therapy. Fifteen CKD-MBD patients undergoing dialysis were enrolled in this study. The patients were orally administered a ferric citrate formulation for 6 months. Their plasma phosphorus concentrations remained unchanged with the switch from other phosphorus adsorbents to the ferric citrate formulation. In addition, the ferric citrate formulation generally allowed for dose reduction of an erythropoiesis stimulating agent with an increased hematopoietic effect. The average values of plasma ferritin level increased after the introduction of a ferric citrate formulation, but did not exceed $100(\mathrm{ng} / \mathrm{mL})$. Interestingly, oxidative stress markers did not increase significantly, and anti-oxidative capacity was not significantly decreased at 6 months after the drug administration. Similarly, no change was observed in any inflammation markers. The ferric citrate formulation induces negligible oxidative stress in CKD-MBD patients receiving dialysis under the present clinical condition.

Key words ferric citrate; phosphate binder; renal anemia; oxidative stress; chronic kidney disease-mineral bone disorder; hemodialysis

The ferric citrate formulation is a new phosphorus adsorbent that, unlike conventional adsorbents, is based on the high affinity that exists between iron and phosphorus and the low solubility of their complex. ${ }^{1,2)}$ The ferric citrate formulation reduces serum phosphorus concentrations in chronic kidney disease-mineral bone disorders (CKD-MBD) patients receiving hemodialysis (HD) in a dose-dependent manner. ${ }^{3)}$ In the phases II and III study conducted in Japan, for example, the serum phosphorus concentration in CKD-MBD patients began to fall at 1 week after administration, and this effect persisted. $^{3-7)}$ In addition, because it is an iron therapeutic, the ferric citrate formulation is also effective in ameliorating anemia in CKD-MBD patient with iron deficiency; when used, it has been reported to result in dose reductions for erythropoiesis stimulating agents (ESA) and intravenous iron preparations. ${ }^{8-10)}$

As stated above, the ferric citrate formulation is expected to be highly beneficial with its multifaceted effects on CKDMBD; however, in general the administration of iron preparations results in increased levels of ferritin, posing concerns about possible organ dysfunction via the induction of oxida-

\footnotetext{
${ }^{\#}$ These authors contributed equally to this work.

tive stress. ${ }^{11-13)}$ In fact, we previously showed that an intravenous iron administration increased oxidative stress in HD patients evaluated by monitoring the redox state of cystein (Cys) 34 in human serum albumin (HSA), ${ }^{14,15)}$ and plasma advanced oxidation protein products (AOPP) level. In the case of the ferric citrate formulation, clinical studies have also found dose-dependent increases in plasma concentrations of iron and ferritin and the values of transferrin saturation (TSAT), posing a concern of oxidative stress. ${ }^{4,5)}$ However, a comprehensive assessment of the effect of the ferric citrate formulation on oxidative stress has not been reported.

In the present study, the effects of the oral administration of a ferric citrate formulation on oxidative stress markers were examined in CKD-MBD patients receiving dialysis therapy under conditions for which the ferric citrate formulation would be therapeutically effective and in which both mineral metabolism and renal anemia would be ameliorated.

\section{MATERIALS AND METHODS}

Materials Ellman's reagent (5-(3-carboxy-4-nitrophenyl)disulfanyl-2-nitrobenzoic acid (DTNB)) was obtained from Dojin Kagaku (Kumamoto, Japan). Other chemicals used in 
this study were of the highest grade commercially available, and all solutions were prepared using deionized and distilled water.

Ethics Statement The study was approved by "Ethics Committee of the Akebono Clinic." All samples were obtained with written informed consent reviewed by the ethical board of the corresponding clinic.

Patients Fifteen stable CKD-MBD patients (11 men and 4 women) receiving dialysis aged 49 to 80 (average $67.1 \pm 10.7$ ) years who had been admitted to the Department of Nephrology of the Akebono Clinic of Japan, between June 2014 and March 2015 were enrolled in this study. At the time of their enrollment, all of the CKD-MBD patients were receiving regular bicarbonate HD therapy (4 to $5 \mathrm{~h}$ per session, 3 times per week) using high-flux polysulfone hollow-fiber dialyzers. Blood samples were drawn from the arterial line at the start of the HD treatment at $0,1,2,3$, and 6 months after the introduction of the ferric citrate formulation. All of the subjects examined in this study have been continued by phosphate binders at least one year at the enrollment and were switched from phosphorus binders in Japan (calcium carbonate $(n=8)$, sevelamer hydrochloride $(n=7)$, bixalomer $(n=2)$ and lanthanum carbonate $(n=2))$ to the ferric citrate formulation. In addition, before switching to the ferric citrate formulation, the administration of an intravenous iron preparation was terminated. Other medications were not changed at least three months before the administration of ferric citrate formulation. The therapeutics and the dialysis conditions were not altered through the study period, except for the ferric citrate formulation and ESA preparations.

Solid Phase Extraction and Electrospray IonizationTime of Flight (ESI-TOF)-MS Measurement of Plasma Samples Blood samples $(9 \mathrm{~mL})$ collected from individual study subjects were immediately diluted with $0.5 \mathrm{M}$ sodium citrate buffer $(\mathrm{pH}$ 4.3) to avoid further oxidation against reduced albumin, as previously reported. ${ }^{16,17)}$ Each plasma fraction was separated by centrifugation $\left(4^{\circ} \mathrm{C}\right.$ for $15 \mathrm{~min}$, $2000 \times \boldsymbol{g})$. After collection, plasma samples were stored at $-80^{\circ} \mathrm{C}$ until analyzed. A $5 \mu \mathrm{L}$ of the above sample was added to $495 \mu \mathrm{L}$ of $50 \mathrm{~mm}$ sodium phosphate buffer ( $\mathrm{pH} 6.0$ ). A solid phase extraction (SPE) column (Bond Elute-C18 EWP $200 \mathrm{mg} / 3 \mathrm{cc}$, Varian, Inc., CA, U.S.A.) was initialized with $10 / 90$ water/acetonitrile containing $0.1 \%$ formic acid, and then equilibrated with water $(1 \mathrm{~mL})$. The above-mentioned diluted plasma sample was applied to the equilibrated SPE column. The column was washed with $10 \%$ acetonitrile $(1.5 \mathrm{~mL})$ containing $0.1 \%$ formic acid, and the albumin was eluted with a $90 \%$ aqueous acetonitrile solution containing $0.1 \%$ formic acid. A $2 \mu \mathrm{L}$ aliquot of the eluent was flow injected into an ESI-TOF-MS (microTOF ${ }^{\circledR}$; Bruker Daltonics Inc., U.S.A.) at a flow rate of $15 \mu \mathrm{L} / \mathrm{min}$ with $10 / 90$ water/acetonitrile containing $0.1 \%$ formic acid using the auto sampler of an Ultimate 3000 (Dionex, Idstein, Germany).

The data were acquired by MicroTOF ${ }^{\circledR}$ software (BrukerDaltonics) and processed for Maxent deconvolution using DataAnalysis ${ }^{\circledR}$ software (Bruker-Daltonics). The deconvolution mass range was set to be from 66000 to $68000 \mathrm{Da}$. The mass peak of the reduced form of HSA and Cys34-cysteinylated HSA (Cys-Cys34-HSA) were automatically assigned and converted to an output text file using script with a resolving power of $10000 \mathrm{~m} / \mathrm{dm}$ and an absolute intensity threshold of
1000. The fraction of Cys-Cys34-HSA (\%) was calculated by (Cys-Cys34-HSA/(Cys-Cys34-HSA+reduced HSA)) $\times 100$ as previously reported. ${ }^{16,17)}$

Measurement of AOPP in Plasma Samples Plasma AOPP levels were determined spectrophotometrically by measuring the absorbance of the samples at $340 \mathrm{~nm}$ and expressed as chloramine-T equivalents after a five-fold dilution of $200 \mu \mathrm{L}$ of plasma with $20 \mathrm{mmol} / \mathrm{L}$ of $\mathrm{pH} 7.4$ phosphate-buffered saline (PBS) and the subsequent addition of $80 \mu \mathrm{L}$ of acetic acid, and reading against a blank PBS solution. ${ }^{18)}$

Measurement of Thiol Contents in Plasma Samples Thiol contents in plasma samples obtained from subjects were estimated by the DTNB method as previously reported. ${ }^{16)}$ Specifically, the increase in absorbance at $405 \mathrm{~nm}$ was monitored against time after the addition of DTNB (final concentration $\left.5.0 \times 10^{-4} \mathrm{M}\right) .{ }^{19)}$

Measurement of Malondialdehyde (MDA) Concentration in Plasma Samples The plasma samples were measured using a TBARS assay kit (Cayman Chemical Company, Ann Arbor, MI, U.S.A.) according to the manufacturer's instructions.

Biochemical Analysis Plasma concentrations of phosphorus, iron $(\mathrm{Fe})$, calcium $(\mathrm{Ca})$, transferrin, ferritin, C-reactive protein (CRP), $\alpha_{1}$-acid glycoprotein (AGP), other standard hematological parameters and biochemical parameters were measured at a contract laboratory (SRL, Inc., Tokyo, Japan).

Statistical Analysis The means for groups were compared by ANOVA followed by Bonferroni multiple comparison. Paired Student's $t$-test was performed to ascertain statistical significance between before or after ferric citrate formulation treatment. All data are expressed as the mean \pm standard deviation (S.D.). Statistical analyses were performed with $\mathrm{R}$ software. A $p$ value $<0.05$ was considered to represent a statistically significant difference.

\section{RESULTS}

Effects of the Ferric Citrate Formulation on Plasma Phosphate Levels and Anemia Parameters In the present study, the effects of switching to the ferric citrate formulation for 6 months on blood phosphorus concentrations were examined in 15 CKD-MBD patients who were already on phosphorus management using phosphorus binders, as mentioned in Materials and Methods. Table 1 provides a summary of the general characteristics of the study participants. Before switching to the ferric citrate formulation, the administration of an intravenous iron preparation $(8 / 15)$ was terminated. $80 \%$ of the patients $(12 / 15)$ were receiving ESA therapy at the beginning of the study. In addition, the regimen of therapeutics and the dialysis conditions were not altered through the study period, except for an adjustment in the dose of the ferric citrate formulation and the ESA preparations. Figure 1 shows the changes in the average dose of ferric citrate formulation during the study period. In this study, all of the subjects were initially treated by a dose of $750(\mathrm{mg} / \mathrm{d})$ of the ferric citrate formulation, and in some subjects, the dose was then increased or decreased depending upon the plasma phosphate levels or blood hemoglobin $(\mathrm{Hb})$ levels.

As shown in Table 2, the plasma phosphorus levels did not change significantly following the 6-month switch from an existing phosphorus adsorbent to the ferric citrate formulation. 
This confirms that the hypophosphatemia effect of the ferric citrate formulation is equivalent to that of other phosphate binders. Similarly, the plasma $\mathrm{Ca}$ concentrations were not largely affected by switching to the ferric citrate formulation (Table 2).

Subsequently, the effects of the administration of the ferric citrate formulation on markers associated with anemia were examined under the same conditions. As shown in Table 2, the blood $\mathrm{Hb}$ level slightly but significantly increased at 6 months after the introduction of the ferric citrate formulation. In contrast, the average dose of ESA was showed the tendency to reduce after the introduction of the ferric citrate formulation (Table 2). In addition, the average plasma concentration of ferritin increased from $49.33 \pm 34.22(\mathrm{ng} / \mathrm{mL})$ before administration to $93.46 \pm 51.69(\mathrm{ng} / \mathrm{mL})$ at 6 months post-dose (Table 2). Among the subjects, three exceeded the $100(\mathrm{ng} / \mathrm{mL})$ plasma ferritin level at the end of the study period (maxima; 253 (ng/ $\mathrm{mL})$ ). The plasma iron levels and TSAT showed a tendency to increase at 6 month after the treatment by ferric citrate formulation (Table 2).

Effects of the Ferric Citrate Formulation on the Markers of Oxidative Stress and Inflammation To clarify whether the ferric citrate preparation enhances oxidative stress to CKD-MBD patients receiving dialysis therapy, we compared to various oxidative stress markers between prior

Table 1. Characteristics of Patient Groups

\begin{tabular}{lc}
\hline \hline & HD patient $(n=15)$ \\
\hline Age (year-old) & $67.1 \pm 10.7$ \\
Gender (M/F) & $11 / 4$ \\
Durations of dialysis (month) & $84.9 \pm 96.7$ \\
Diabetes/non-diabetes & $6 / 9$ \\
Plasma albumin (g/dL) & $3.7 \pm 0.3$ \\
Plasma uric acid (mg/dL) & $7.2 \pm 1.3$ \\
AST (IU/L) & $10.2 \pm 3.6$ \\
ALT (IU/L) & $9.8 \pm 4.7$ \\
BUN (mg/dL) & $63.1 \pm 6.9$ \\
Plasma creatinine (mg/dL) & $12.0 \pm 2.5$ \\
\hline
\end{tabular}

Values are expressed as the mean \pm S.D.

Table 2. Effects of the Ferric Citrate Formulation on Mineral and Anemia Parameters

\begin{tabular}{lcc}
\hline \hline & $\begin{array}{c}\text { Before the ferric } \\
\text { citrate formulation } \\
\text { administration } \\
(n=15)\end{array}$ & $\begin{array}{c}\text { Six month after the } \\
\text { ferric citrate } \\
\text { formulation } \\
\text { administration } \\
(n=15)\end{array}$ \\
\hline Mineral parameters & $6.08 \pm 1.42$ & $5.91 \pm 0.67$ \\
Plasma phosphorus (mg/dL) & $8.92 \pm 0.43$ & $9.05 \pm 0.47$ \\
Plasma calcium (mg/dL) & & \\
Anemia parameters & $10.39 \pm 0.80$ & $11.04 \pm 1.48^{*}$ \\
Hb (g/dL) & $3357.14 \pm 1029.33$ & $3136.36 \pm 1704.27$ \\
ESA dose (IU/week) & $49.33 \pm 34.22$ & $93.46 \pm 51.67 * *$ \\
Plasma ferritin $(\mathrm{ng} / \mathrm{mL})$ & $61.95 \pm 31.57$ & $71.44 \pm 17.60$ \\
Plasma iron $(\mu \mathrm{g} / \mathrm{dL})$ & $26.46 \pm 12.30$ & $31.01 \pm 7.23$ \\
TSAT $(\%)$ &
\end{tabular}

Values are expressed as the mean \pm S.D. $* p<0.05$ as compared with before the ferric citrate formulation administration. $* * p<0.01$ as compared with before the ferric citrate formulation administration. to and after the use of the ferric citrate formulation. Thus, the time dependent changes in the Cys-Cys34-HSA fraction were examined after the administering the ferric citrate preparation to CKD-MBD patients. Figures $2 \mathrm{~A}-\mathrm{C}$ show typical MS charts obtained from healthy subjects and at 0 and 6 months after the administration of the ferric citrate formulation to CKD-MBD patients with dialysis. Compared to healthy subjects, the peak for Cys-Cys34-HSA was increased and that for the reduced form of Cys34 was decreased in the CKD-MBD patients, indicating increased oxidative stress. As shown in Fig. 2D, the Cys-Cys34-HSA fraction did not change for periods of up to 4 months after the introduction of the ferric citrate preparation, while after that, the Cys-Cys34-HSA fraction began to show the tendency to reduce. As compared to before the administration of the ferric citrate formulation, the values of the Cys-Cys34-HSA fraction for most of the patients (13/15) were reduced at the end of the study period, including subjects for whom the ferritin levels exceeded 100 (ng/mL) (Fig. 2E).

Subsequently, the plasma thiol content, an indicator of anti-oxidant capacity in plasma, was examined because if reactive oxygen species were to have been produced by the action of the iron formulation, the plasma thiol content would decrease by eliminating the reactive oxygen species that were produced. As shown in Fig. 3A, the plasma thiol content was unchanged for periods of 4 months after the introduction of a ferric citrate preparation, and after this interval, it became to show the tendency to increase. As compared to before the administration of a ferric citrate formulation, most of the pa-

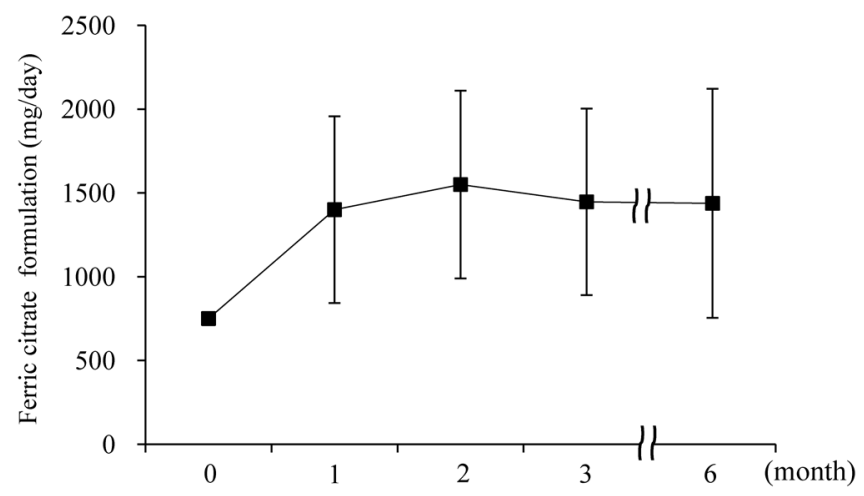

Fig. 1. Changes in Average Daily Doses (mg/d) of the Ferric Citrate Formulation

Values are expressed as the mean \pm S.D.; $n=15$ patients for each month.

Table 3. Effects of the Ferric Citrate Formulation on Oxidative and Inflammation Parameters

\begin{tabular}{ccc}
\hline \hline & $\begin{array}{c}\text { Before the ferric } \\
\text { citrate formulation } \\
\text { administration } \\
(n=15)\end{array}$ & $\begin{array}{c}\text { Six month after the } \\
\text { ferric citrate } \\
\text { formulation } \\
\text { administration } \\
(n=15)\end{array}$ \\
\hline Oxidative stress markers & & $151.02 \pm 46.30$ \\
Plasma AOPP $(\mu \mathrm{M})$ & $145.78 \pm 37.64$ & $14.08 \pm 9.66$ \\
Plasma MDA $(\mu \mathrm{M})$ & $16.07 \pm 10.84$ & $0.16 \pm 0.25$ \\
Inflammation markers & $0.28 \pm 0.65$ & $123.68 \pm 78.67$ \\
Plasma CRP $(\mathrm{mg} / \mathrm{dL})$ & $126.28 \pm 105.89$ & \\
Plasma AGP $(\mathrm{mg} / \mathrm{dL})$ &
\end{tabular}

Values are expressed as the mean \pm S.D. 
(A)

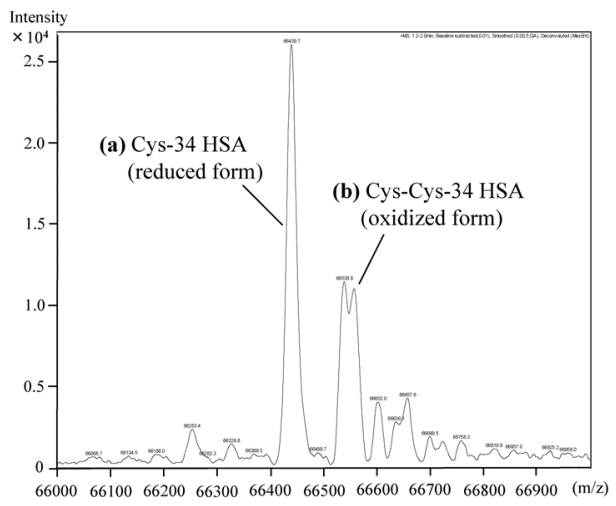

(C)

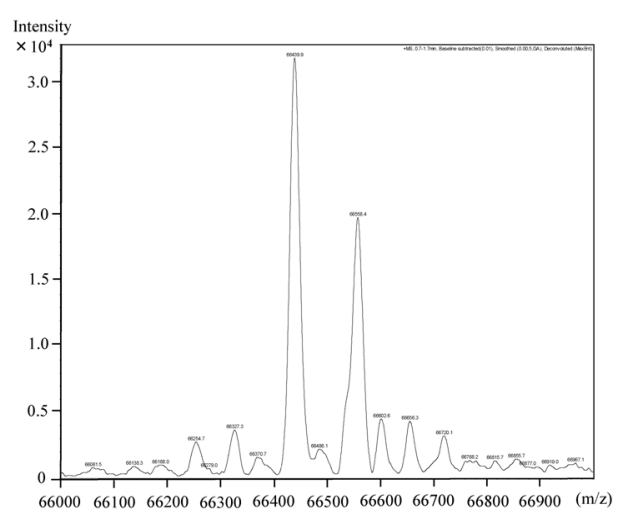

(E)
(B)

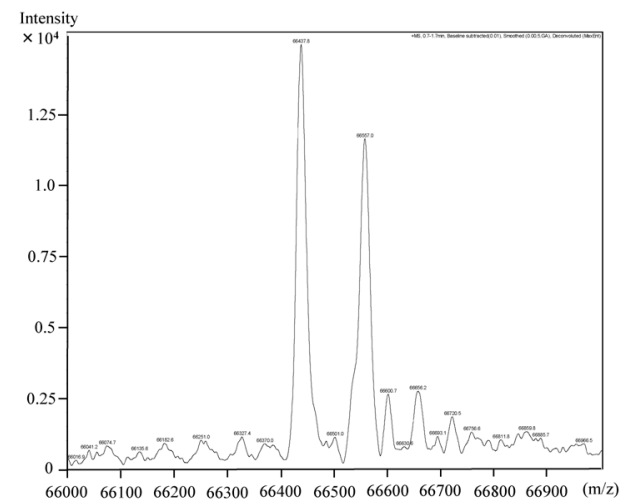

(D)

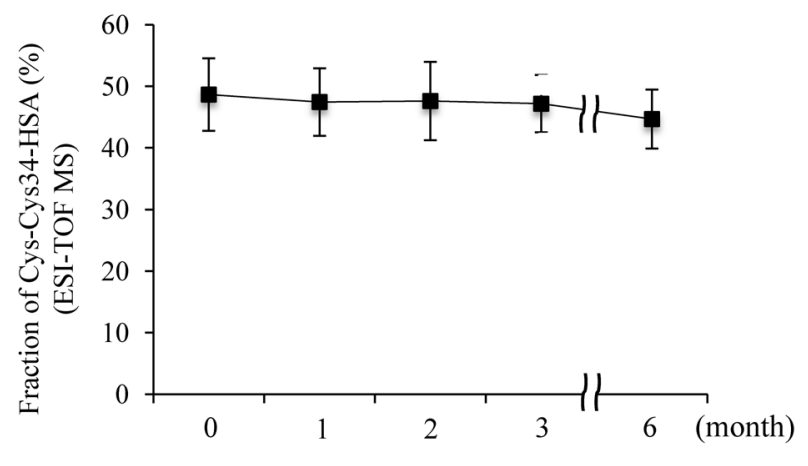

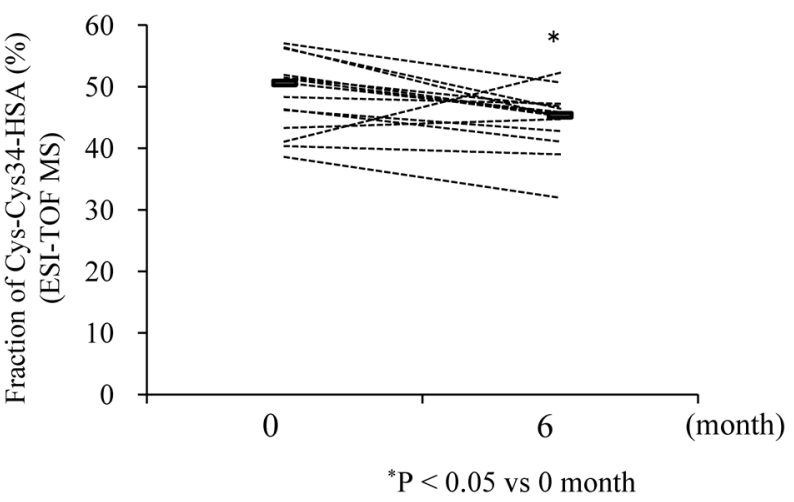

Fig. 2. Representative Deconvoluted ESI-TOF-MS Spectra of HSA and the Amount of the Cys-Cys34-HSA Fraction; (A) Spectrum of HSA from a Healthy Subject, (B) Spectrum of HSA from Patients at the Beginning of the Study, (C) Spectrum of HSA from Patients after a Ferric Citrate Treatment for 6 Months, (D) The Cys-Cys34-HSA Fraction during the Study Period, (E) The Comparison of Cys-Cys34-HSA Fraction between Beginning and End of the Study

Values are expressed as the mean \pm S.D.; $n=15$ patients for each month. $* p<0.05$ as compared with 0 month.

tients $(11 / 15)$ had an elevated thiol content at the end of the study period, including subjects for whom the plasma ferritin levels exceeded $100(\mathrm{ng} / \mathrm{mL})$ (Fig. 3B). This change is in good agreement with the results for the Cys-Cys34-HSA fraction.

To further confirm whether ferric citrate formulation did not induced oxidative stress, plasma concentrations of AOPP, a marker for oxidative damage of proteins and MDA, a marker for lipid oxidation, were measured before introduction of ferric citrate formulation and over a period of days. Plasma AOPP and MDA concentrations were not significantly altered 
(A)

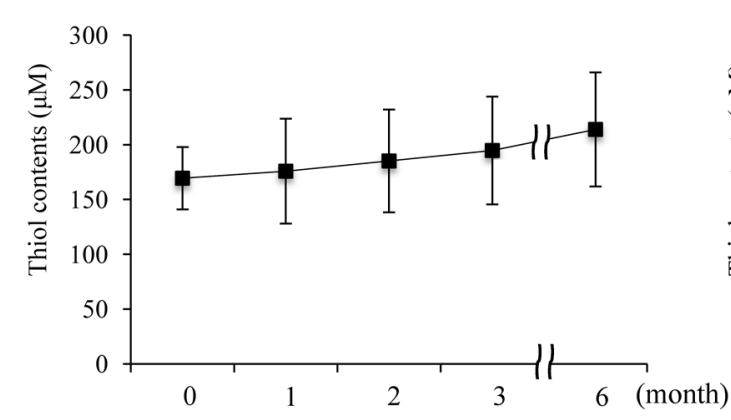

(B)

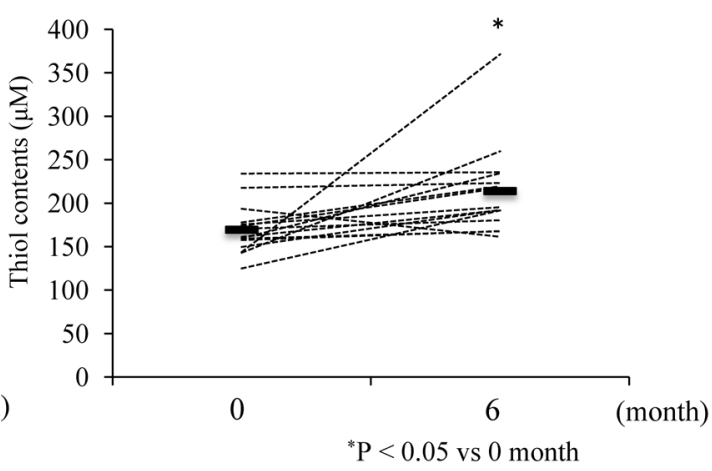

Fig. 3. Effects of the Ferric Citrate Formulation on the Plasma Thiol Contents (\%)

Values are expressed as the mean \pm S.D.; $n=15$ patients for each month. ${ }^{*} p<0.05$ as compared with 0 month.

after the switching to the ferric citrate formulation for 6 months (Table 3).

The effects of a ferric citrate formulation on the plasma concentrations of inflammation markers, CRP and AGP (also referred to as orosomucoid) were examined. As shown in Table 3, the results revealed no significant change in any of the inflammation markers after the administration of a ferric citrate formulation for 6 months.

\section{DISCUSSION}

The objective of the present study was to examine whether the administration of a ferric citrate formulation, an oral phosphorus adsorbent, induces oxidative stress in CKD-MBD patients receiving dialysis. The findings indicate that oxidative stress is not enhanced under conditions at which a ferric citrate formulation is therapeutically effective.

Clinical trials have clearly revealed that the ferric citrate formulation is characterized by two therapeutic features: 1) suppression of phosphorus accumulation in the plasma, 2) amelioration of renal anemia with iron deficiency by iron supplementation. The improvement of renal anemia is particularly unique to the ferric citrate formulation because it is not found with any other phosphorus adsorbent. ${ }^{8-10)}$ A post-marketing clinical investigation conducted also confirmed the aforementioned two effects in CKD-MBD patients who were undergoing hemodialysis for periods of up to 6 months.

While the ferric citrate formulation ameliorates anemic states by virtue of its iron supplementation effect, the possible induction of oxidative stress via increased hydroxyl radical production due to the Fenton reaction is an issue of great concern. In fact, the administration of an intravenous iron preparation has been reported to increase the oxidation of plasma proteins, particularly albumin (oxidative modification of Cys34, increased carbonyl content, and AOPP production) and the production of lipid peroxides and oxidized forms of nucleic acids. ${ }^{14,15,20-24)}$ Such induction of oxidative stress associated with the administration of an iron formulation causes vascular endothelial dysfunction, thus increasing the risk of cardiovascular disease. ${ }^{11-13)}$ In the case of the ferric citrate formulation as well, increases in the plasma ferritin levels and TSAT associated with its administration were found in clinical studies, ${ }^{4,5)}$ and its influence on oxidative stress is, therefore, a subject of concern.

To obtain evidence for these issues, the present study was conducted to measure oxidative stress markers and the antioxidant capacity of plasma of CKD-MBD patients receiving dialysis before and at 6 months after the administration of an ferric citrate formulation. The findings indicate increases in oxidative stress markers are absent and anti-oxidative capacity parameters are decreased. These findings indicate that the administration of the ferric citrate preparation does not enhanced oxidative stress, and that the anti-oxidant defense system in the plasma is not impaired. This was supported by the fact that inflammatory reactions associated with oxidative stress were not detected. Terawaki et al. examined the redox state of Cys34 in HSA in various dialysis patients, and reported that the incidence of serious cardiovascular diseases increased with decreasing concentrations of reduced HSA. ${ }^{25-27)}$ In addition, Lim et al. found that the redox state of HSA is a positive predictor of mortality in normoalbuminemic HD patients. ${ }^{28)}$ Similarly, Jalan et al. found that the 1-year survival rate for liver cirrhosis patients could be predicted by the concentration of reduced HSA in their plasma. ${ }^{29)}$ Taking into consideration the fact that modified albumin molecules lack multiple functions, Domenicali et al. proposed that the concentration of reduced HSA should be measured to indicate the "effective albumin concentration" as a new indicator in lieu of the conventional parameter total albumin concentration. ${ }^{29)}$ In the present study, the concentration of reduced HSA was not decreased with the administration of the ferric citrate formulation; therefore, there was no reduction in the effective albumin concentrations (from $1.92 \pm 0.22$ to $2.09 \pm 0.27$ (g/dL)), suggesting that the administration of the ferric citrate formulation is unlikely to increase the risk of cardiovascular disease via the induction of oxidative stress. This is further supported by the fact that, unlike an intravenous iron injection, the oral administration of the ferric citrate formulation did not increase AOPP production because it is known that the AOPP levels increase with the intravenous iron administration, and this change is reportedly associated with the carotid artery intima-media thickness, a marker of early atherosclerosis. ${ }^{21,24)}$

The mechanism by which, in contrast to intravenous iron administration, the ferric citrate formulation did not increase the oxidative stress is not clear based on the present limited data. One possible reason for this is the difference in the form 
of iron that is present in plasma between two preparations. When iron is administrated orally, it gradually enters the blood stream, and is subsequently present in a protein-bound form, especially bound to transferrin and albumin, while an intravenous iron injection results in the rapidly appearance of iron in the blood circulation, and, as a result, both protein bound and unbound forms are present, due to the saturation of binding. Such unbound iron molecules could enhance the level of oxidative stress via the production of reactive oxygen species. In fact, oxidative stress markers have been shown to increase persistently with a 3 times-a-week administration for 1 month, whereas, in the case of a once-a-week administration for 3 months, oxidative stress markers are suppressed, despite the constant total dose of intravenous iron for both regimens; the contributions of different free iron levels based on differences in dosing frequency are likely. ${ }^{15}$ )

In the present study, the Cys-Cys34-HSA fraction and plasma thiol content show a tendency to reduce and to increase, respectively, especially at 6 months after the administration of ferric citrate formulation, suggesting the slight reduction of oxidative stress. Based on the present limited data, it is not possible to explain these unexpected phenomena. Since alternation of above parameters started to several months later after the administration of ferric citrate formulation, it suggest that the slight reduction of oxidative stress might be an indirect effect of ferric citrate formulation rather than its direct effect. However, it must to be carefully to interpret the ferric citrate formulation induced the slight reduction of oxidative stress because other oxidative stress markers did not significantly changed during a study period. This might be figured out by our ongoing study that examine whether oxidative stress induced at 12 months after the introduction of ferric citrate formulation.

It should be noted, however, that the present study was subject to limitations due to the absence of a control group as it was a single-site observational study involving limited patient numbers. Furthermore, since the present investigation was a 6 month observation, it would be interesting to know whether the administration of a ferric citrate formulation for more than 6 months would have an effect on the level of oxidative stress. To resolve these issues, it will be necessary to conduct a randomized control trial with a larger sample size and a longer period in future.

\section{CONCLUSION}

In the present study, the effects of a ferric citrate formulation as a new phosphorus adsorbent on oxidative stress in the general circulation was examined in CKD-MBD patients in clinical practice settings. Taking into account the findings comprehensively, it is demonstrated, for the first time, that the administration of the ferric citrate formulation for 6 months does not largely influence oxidative stress in CKD-MBD patients receiving dialysis under the present clinical condition.

Acknowledgment This work was supported by the Research Grant of Japanese Association of Dialysis Physicians.

Conflict of Interest The authors declare no conflict of interest.

\section{REFERENCES}

1) Lewis JB, Sika M, Koury MJ, Chuang P, Schulman G, Smith MT, Whittier FC, Linfert DR, Galphin CM, Athreya BP, Nossuli AK, Chang IJ, Blumenthal SS, Manley J, Zeig S, Kant KS, Olivero JJ, Greene T, Dwyer JP, Collaborative Study Group. Ferric citrate controls phosphorus and delivers iron in patients on dialysis. $\mathrm{J}$. Am. Soc. Nephrol., 26, 493-503 (2015).

2) Nastou D, Fernández-Fernández B, Elewa U, González-Espinoza L, González-Parra E, Sanchez-Niño MD, Ortiz A. Next-generation phosphate binders: focus on iron-based binders. Drugs, 74, 863-877 (2014).

3) Yokoyama K, Hirakata H, Akiba T, Sawada K, Kumagai Y. Effect of oral JTT-751 (ferric citrate) on hyperphosphatemia in hemodialysis patients: results of a randomized, double-blind, placebocontrolled trial. Am. J. Nephrol., 36, 478-487 (2012).

4) Yokoyama K, Akiba T, Fukagawa M, Nakayama M, Sawada K, Kumagai Y, Chertow GM, Hirakata H. A randomized trial of JTT-751 versus sevelamer hydrochloride in patients on hemodialysis. Nephrol. Dial. Transplant., 29, 1053-1060 (2014).

5) Yokoyama K, Hirakata H, Akiba T, Fukagawa M, Nakayama M, Sawada K, Kumagai Y, Block GA. Ferric citrate hydrate for the treatment of hyperphosphatemia in nondialysis-dependent CKD. Clin. J. Am. Soc. Nephrol., 9, 543-552 (2014).

6) Yokoyama K, Akiba T, Fukagawa M, Nakayama M, Hirakata H. JTT-751 for treatment of patients with hyperphosphatemia on peritoneal dialysis. Nephron Clin. Pract., 128, 135-140 (2014).

7) Yokoyama K, Akiba T, Fukagawa M, Nakayama M, Sawada K, Kumagai Y, Chertow GM, Hirakata H. Long-term safety and efficacy of a novel iron-containing phosphate binder, JTT-751, in patients receiving hemodialysis. J. Ren. Nutr., 24, 261-267 (2014).

8) Block GA, Fishbane S, Rodriguez M, Smits G, Shemesh S, Pergola PE, Wolf M, Chertow GM. A 12-week, double-blind, placebo-controlled trial of ferric citrate for the treatment of iron deficiency anemia and reduction of serum phosphate in patients with CKD Stages 3-5. Am. J. Kidney Dis., 65, 728-736 (2015).

9) Nakanishi T, Hasuike Y, Nanami M, Yahiro M, Kuragano T. Novel iron-containing phosphate binders and anemia treatment in CKD: oral iron intake revisited. Nephrol. Dial. Transplant., Jul 3, pii: gfv268 (2015). [Epub ahead of print].

10) Feldman HI, Santanna J, Guo W, Furst H, Franklin E, Joffe M, Marcus S, Faich G. Iron administration and clinical outcomes in hemodialysis patients. J. Am. Soc. Nephrol., 13, 734-744 (2002).

11) Rooyakkers TM, Stroes ES, Kooistra MP, van Faassen EE, Hider RC, Rabelink TJ, Marx JJ. Ferric saccharate induces oxygen radical stress and endothelial dysfunction in vivo. Eur. J. Clin. Invest., 32 (Suppl. 1), 9-16 (2002).

12) Bishu K, Agarwal R. Acute injury with intravenous iron and concerns regarding long-term safety. Clin. J. Am. Soc. Nephrol., 1 (Suppl. 1), S19-S23 (2006)

13) Kletzmayr J, Hörl WH, Kletzmayr J, Hörl WH. Iron overload and cardiovascular complications in dialysis patients. Nephrol. Dial. Transplant., 17 (Suppl. 2), 25-29 (2002).

14) Anraku M, Kitamura K, Shinohara A, Adachi M, Suenaga A, Maruyama T, Miyanaka K, Miyoshi T, Shiraishi N, Nonoguchi H, Otagiri M, Tomita K. Intravenous iron administration induces oxidation of serum albumin in hemodialysis patients. Kidney Int., 66 , 841-848 (2004).

15) Anraku M, Kitamura K, Shintomo R, Takeuchi K, Ikeda H, Nagano J, Ko T, Mera K, Tomita K, Otagiri M. Effect of intravenous iron administration frequency on AOPP and inflammatory biomarkers in chronic hemodialysis patients: A pilot study. Clin. Biochem., 41, 1168-1174 (2008).

16) Nagumo K, Tanaka M, Chuang VT, Setoyama H, Watanabe H, Yamada N, Kubota K, Tanaka M, Matsushita K, Yoshida A, Jinnouchi H, Anraku M, Kadowaki D, Ishima Y, Sasaki Y, Otagiri 
M, Maruyama T. Cys34-cysteinylated human serum albumin is a sensitive plasma marker in oxidative stress-related chronic diseases. PLoS ONE, 9, e85216 (2014)

17) Kubota K, Nakayama A, Takehana K, Kawakami A, Yamada N, Suzuki E. A simple stabilization method of reduced albumin in blood and plasma for the reduced/oxidized albumin ratio measurement. Int. J. Biomed. Sci., 5, 293-301 (2009).

18) Witko-Sarsat V, Friedlander $M$, Capeillère-Blandin C, NguyenKhoa T, Nguyen AT, Zingraff J, Jungers P, Descamps-Latscha B. Advanced oxidation protein products as a novel marker of oxidative stress in uremia. Kidney Int., 49, 1304-1313 (1996).

19) Anraku M, Takeuchi K, Watanabe H, Kadowaki D, Kitamura K, Tomita K, Kuniyasu A, Suenaga A, Maruyama T, Otagiri M. Quantitative analysis of cysteine-34 on the anitioxidative properties of human serum albumin in hemodialysis patients. J. Pharm. Sci., 100, 3968-3976 (2011).

20) Garneata L. Intravenous iron, inflammation, and oxidative stress: is iron a friend or an enemy of uremic patients? J. Ren. Nutr., 18, 40-45 (2008)

21) Witko-Sarsat V, Friedlander MA, Nguyen Khoa T, Capeillère-Blandin C, Nguyen AT, Canteloup S, Dayer JM, Jungers P, Drüeke T, Descamps-Latscha B. Advanced oxidation protein products (AOPP) as novel mediators of inflammation and monocyte activation in chronic renal failure. J. Immunol., 161, 2524-2532 (1998).

22) Maruyama Y, Nakayama M, Yoshimura K, Nakano H, Yamamoto $\mathrm{H}$, Yokoyama K, Lindholm B. Effect of repeated intravenous iron administration in haemodialysis patients on serum 8-hydroxy-2'deoxyguanosine levels. Nephrol. Dial. Transplant., 22, 1407-1412 (2007).

23) Yoshimura K, Nakano H, Yokoyama K, Nakayama M. High iron storage levels are associated with increased DNA oxidative injury in patients on regular hemodialysis. Clin. Exp. Nephrol., 9, 158-163 (2005)

24) Drüeke T, Witko-Sarsat V, Massy Z, Descamps-Latscha B, Guerin AP, Marchais SJ, Gausson V, London GM. Iron therapy, advanced oxidation protein products, and carotid artery intima-media thickness in end-stage renal disease. Circulation, 106, 2212-2217 (2002).

25) Terawaki H, Matsuyama Y, Matsuo N, Ogura M, Mitome J, Hamaguchi A, Terada T, Era S, Hosoya T. A lower level of reduced albumin induces serious cardiovascular incidence among peritoneal dialysis patients. Clin. Exp. Nephrol., 16, 629-635 (2012).

26) Terawaki H, Era S, Nakayama M, Hosoya T. Decrease in reducedform albumin among chronic kidney disease patients: new insights in cardiovascular complications. Ther. Apher. Dial., 15, 156-160 (2011).

27) Matsuyama $Y$, Hayashi $T$, Terawaki H, Negawa T, Terada T, Okano Y, Era S. Human astrocytes and aortic endothelial cells actively convert the oxidized form of albumin to the reduced form: reduced albumin might participate in redox regulation of nerve and blood vessel systems. J. Physiol. Sci., 59, 207-215 (2009).

28) Lim PS, Jeng Y, Wu MY, Pai MA, Wu TK, Liu CS, Chen CH, Kuo YC, Chien SW, Chen HP. Serum oxidized albumin and cardiovascular mortality in normoalbuminemic hemodialysis patients: a cohort study. PLoS ONE, 8, e70822 (2013).

29) Domenicali M, Baldassarre M, Giannone FA, Naldi M, Mastroroberto M, Biselli M, Laggetta M, Patrono D, Bertucci C, Bernardi M, Caraceni P. Posttranscriptional changes of serum albumin: clinical and prognostic significance in hospitalized patients with cirrhosis. Hepatology, 60, 1851-1860 (2014). 